\title{
Visual memory and visual perception: when memory improves visual search
}

\author{
Benoit Riou • Mathieu Lesourd • Lionel Brunel • \\ Rémy Versace
}

Published online: 5 February 2011

(C) Psychonomic Society, Inc. 2011

\begin{abstract}
This study examined the relationship between memory and perception in order to identify the influence of a memory dimension in perceptual processing. Our aim was to determine whether the variation of typical size between items (i.e., the size in real life) affects visual search. In two experiments, the congruency between typical size difference and perceptual size difference was manipulated in a visual search task. We observed that congruency between the typical and perceptual size differences decreased reaction times in the visual search (Exp. 1), and noncongruency between these two differences increased reaction times in the visual search (Exp. 2). We argue that these results highlight that memory and perception share some resources and reveal the intervention of typical size difference on the computation of the perceptual size difference.
\end{abstract}

Keywords Embodied memory $\cdot$ Perception $\cdot$ Visual search

Embodied theory argues that memory is based on sensorymotor traces that can be activated when a part of the encoded stimulus or situation is processed. Consequently, our memory should be the result of our own perception (e.g., Barsalou,

\author{
B. Riou $(\triangle) \cdot$ M. Lesourd $\cdot$ L. Brunel $\cdot$ R. Versace \\ Laboratoire d'Etude des Mécanismes Cognitifs (EMC), EA 3082, \\ Université Lumière Lyon 2, \\ 5, avenue Pierre Mendès France, \\ 69676 Bron Cedex, France \\ e-mail: benoit.riou@univ-lyon2.fr \\ M. Lesourd \\ e-mail: mathieu.lesourd@univ-lyon2.fr \\ L. Brunel \\ e-mail: lionel.brunel@univ-lyon2.fr \\ R. Versace \\ e-mail: remy.versace@univ-lyon2.fr
}

1999, 2003, 2005; for a review, see Barsalou, 2008). On the basis of their work in this field, Versace, Labeye, Badard, and Rose (2009) proposed that memory is distributed across the neuronal networks involved in sensory-motor processing. Brain-imaging studies have provided a wide range of arguments supporting this hypothesis (Howard et al., 1998; Martin, Haxby, Lalonde, Wiggs, \& Ungerleider 1995; Paulesu et al., 1995; for a review, see Martin \& Chao, 2001). These studies have indicated that sensory-motor areas of the brain, which are not involved in the perceptual processing of a stimulus, are activated when participants perform a memory task. In line with the imaging studies, behavioural studies have demonstrated the modality-specific character of memory (Barsalou, Pecher, Zeelenberg, Simmons, \& Hamann 2005; Pecher, Zeelenberg \& Barsalou 2003). In other words, each form of knowledge (i.e., conceptual or episodic) is sensory-motor dependent. Pecher et al. concluded that the cost observed in a property verification task associated with a concept involving six different modalities (vision, audition, touch, taste, smell, and action) was due to the change of the property modality from one trial to the next. The same kind of switch cost was observed during perceptual processing (Spence, Nicholls, \& Driver 2001). Thus, it seems that our memory is created during our perceptual experience and consists of sensory-motor memory traces.

Moreover, a variety of recent results have shown that perception and memory processes seem to overlap. Vermeulen, Corneille, and Niedenthal (2008) demonstrated that memory may rely on the same sensory-motor systems that were involved in the original perception of the stimulus. For example, in a high-load condition (HLC), three items (all of which could be visual or auditory) were presented before a property verification 
task (visual or auditory). After the participants had performed the property verification task, the same three or another three items were presented. In this phase, the participants had to decide whether the three items were the same or different. The results revealed longer reaction times (RTs) for both the property verification task and recognition when both the memory load and the property verification task involved the same modality rather than different ones. Van Dantzig, Pecher, Zeelenberg and Barsalou (2008) showed that perception influences memory processes. Their results revealed a modality-switch effect across perceptual processing and conceptual processing. In their study, participants had to decide whether a perceptual stimulus (in three modalities: audition, touch, or vision) appeared on the right or the left side of the screen. Immediately after this, they performed a property verification task. The RTs were longer when the modality of the perceptual stimulus and the modality involved in the property verification task were different rather than the same. The authors concluded that both perceptual and conceptual processing seem to be based on the same distributed system (see also Vallet, Brunel, \& Versace 2010; Vermeulen, Mermillod, Godefroid, \& Corneille 2009). In such cases, memory activation seems to involve the same brain regions that are involved during perceptual processing (for a review, see Martin \& Chao, 2001). In consequence, it might be possible to predict that this activation would occur in all memory processes (i.e., conscious memory processes such as mental imagery, see Martin et al. (1995), or nonconscious memory processes such as categorization, see Slotnick \& Schacter (2006)) and at an early stage of brain functioning.

The sensory-motor dimensions involved during the experience of stimuli would be automatically activated during a memory task. We have presented several examples supporting the idea that whether it is perceived or activated in memory, a given sensory dimension could influence memory, and also perhaps perceptual, tasks (see Hansen, Olkkonen, Walter, \& Gegenfurtner 2006). According to this hypothesis, there is an overlap between memory and perception. These two cognitive functions may share cerebral space, resources, and common processes (Slotnick, 2004; Weinberger, 2004). The reactivated sensory-motor dimensions may influence a perceptual task, and thus the activation of the sensory-motor dimension during memory processing may influence the performance on a perceptual task in the same sensory-motor dimension. This hypothesis was partly tested by Brunel, Labeye, Lesourd, and Versace (2009). The authors showed that the reactivation of a memory dimension influences a perceptual task. Using a short-term-priming paradigm with two distinct phases-a learning phase, consisting of the association between a geometrical shape and a white noise, and a priming phase, examining the priming effect of the geometrical shape on the discrimination of two isolated target tones - the authors found that only the prime previously associated with the sound in the learning phase had a priming effect on the processing of the target. However, in this study, the memory activation occurred before and not during the perceptual task.

The aim of the present work was to reveal the influence of the activation of visual memory features (or dimensions) on an ongoing perceptual task. In particular, we wanted to show that the manipulation of a typical size difference-which is not perceptually present (typical size of objects) — between a target and distractors could influence accuracy on the perceptual task in progress. We therefore used a classical visual search paradigm (see Treisman \& Gormican, 1988) in which the targets and distractors differed on a physical dimension, namely their physical size (the size of the images displayed on the screen). In our experiments, we asked the participants to identify whether a picture (representing a familiar object) was physically (i.e., perceptually) larger (Exp. 1) or smaller (Exp. 2) in size than other pictures displayed on the screen (expressed in terms of the number of pixels). In other words, the target, which was always a picture of a large object (typical size), could vary in terms of its (physical) size on the screen. The distractors, which always had the same size on the screen, could vary in terms of their typical size (pictures of typically large or small objects; e.g., a wardrobe or a whistle). Two conditions were therefore possible: In Experiment 1, the typical size difference, where present, could only be the same as (congruent to) the size difference on screen; in Experiment 2, the typical size difference could only be different from (noncongruent to) the size difference on screen.

As argued above, memory and perception processes seem to be based on the same system or to share resources. Consequently, these two functions should reciprocally influence one another. Our main hypothesis was that the typical size of objects is automatically simulated during perception. In these conditions, we should observe two reverse effects, depending on whether a congruent or noncongruent typical size difference was added. The typical target size was chosen in such a way that the targets were always large objects. Consequently, (1) when the distractors had a large typical size, no typical size difference existed; (2) when the distractors had a small typical size, there was indeed a typical size difference. If the typical size of objects is automatically activated during the perceptual task, in Experiment 1 the congruency between the typical and perceptual size differences should lead to faster responses in the visual search task. In contrast, in Experiment 2 , the noncongruency between the typical and perceptual size differences should lead to slower responses in the visual search task. In line with Treisman and Gormican (1988), we also predicted that the effects should become more pronounced as the number of distractors increased. 
In Experiment 1, we further assumed that the effect of the typical size difference should cause the participants to answer "present" when the physical size difference was either present or absent (because the typical size difference was congruent with the visual search task). In Experiment 2 , the effect of the typical size difference should cause the participants to answer "absent" when the physical size difference was present, but no effect of typical size difference should be observed when the physical size difference was absent (because the typical size difference was not congruent with the visual search task).

\section{Experiment 1}

Method

\section{Participants}

Thirty-six right-handed volunteer participants were recruited for the experiment. All of them were students at the Université Lumière Lyon 2, France, and had normal or corrected-to-normal vision.

\section{Stimuli}

Thirty pictures of manufactured objects were used. The stimuli belonged to the laboratory's database and had already been used in other experiments.

Typical size difference Twenty of the objects had a large typical size, and the 10 others had a small typical size. Small typical size defined objects smaller than $20 \mathrm{~cm}$ in real life. Large typical size defined objects larger than $1 \mathrm{~m}$ but less than $3 \mathrm{~m}$ in real life. These limits in the choice of size prevented any excessive disparity in typical size between items. For instance, a large object could be judged as small if distractors even bigger than itself surrounded it. Half of the typically large items were used as targets and the other half as distractors. The target items for half of the participants served as distractors for the other half. The participants did not know the pictures used as targets, with the result that objects were not task relevant. The distractors could have a large typical size, as the target did, or be smaller than the target. If the distractors had a small typical size, then typical size difference was present. If the distractors had a large typical size, then typical size difference was absent.

Physical size difference The participants had to detect whether one picture (target) was displayed larger than the others pictures (distractors) on the screen. The target could be displayed either as large as the distractors ( 80 pixels) or larger than them (130 pixels). ${ }^{1}$ The 80 - or 130 -pixel size was measured along the largest length of an object (width or height). The other dimension was variable, in order to keep the object's proportions and to prevent any distortion of the objects during dimensioning. In order to control for the area occupied by each picture on the screen, items that were too tapered (in width or height) were excluded. In this way, the area occupied by the pictures on the screen in each physical size condition (80 or 130 pixels) was relatively constant. All of the pictures were presented as greyscale images in order to avoid any colour-related bias. If the target was displayed larger than the distracters, then the physical size difference is present. If the target was displayed as large as the distracters, then the physical size difference is absent.

It should be noted that the typical target size was still large, and the physical size on screen could be 130 or 80 pixels. The typical size of the distractors could be either large or small, but their physical size on screen was always 80 pixels.

Displays The distribution of the stimuli on the screen was determined by dividing the screen into a matrix of 36 positions $(6 \times 6$ cells $)$. From these, 28 positions were selected. The 4 extreme positions in each corner, which were too far from the centre of the visual field, and the 4 most central positions, which were too close to the fixation point, were eliminated. Different displays were created randomly for each of the ten different targets as a function of the number of items (two, six, or ten), leading to 30 different displays.

Each target picture was repeated 12 times, according to the 12 experimental conditions. Each target picture was assigned to a specific display location, such that two different target pictures could not be displayed in a same location. A given target picture always had the same display location across the 12 experimental conditions (see Fig. $1 \mathrm{a}$ and $\mathrm{b}$ for the six-item conditions with physical size difference present). In this way, a comparison of the conditions with physical size difference present and absent would record only the typical size difference between the target and the distractors (present or absent), all other things being equal.

Two intralist blocks of 60 trials were defined. For the same specific combination of physical size difference and number of items (e.g., physical size difference present among six items), the two typical size conditions was distributed across the two blocks (e.g., typical size difference present in Block 1, absent in Block 2). A semirandom order was applied to the trials within each block to ensure that the targets differed between trials. As a result, the location of the target could not be predicted from one trial to the next.

\footnotetext{
${ }^{1}$ The difference in physical size between the target and the distractors was chosen during a pilot study to ensure that the participants used serial search to perform the task
} 
Fig. 1 (a) Example of a display of six items in Experiment 1, when typical size difference was absent and physical size difference was present. Clockwise from the left, the objects are a pinball machine, bicycle, slide, oven, bunk bed, and desk. (b) Example of a display of six items when typical size difference was present and physical size difference was also present. Clockwise from the left, the objects are a baby bottle, whistle, clock, pipe, set of pliers, and desk

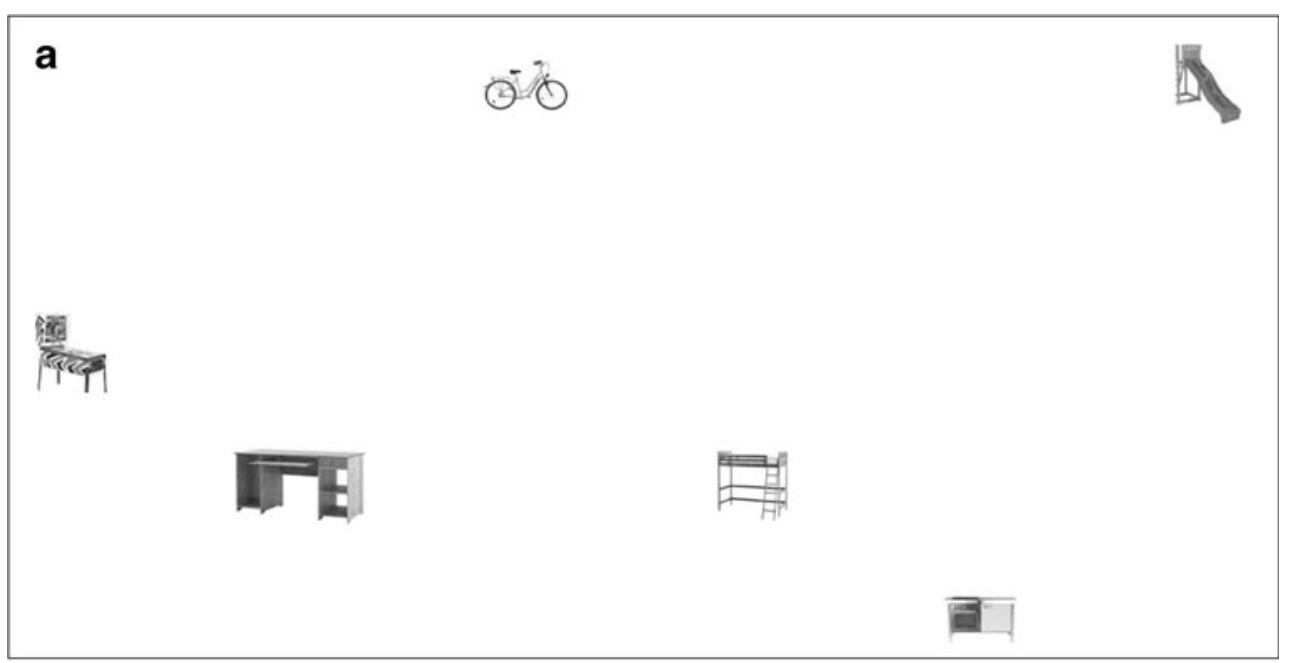

b
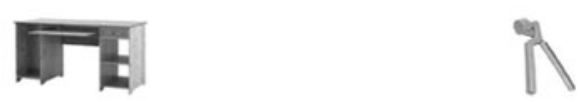

The experiment had a 3 (number of items: two, six, or ten) x 2 (physical size difference: present or absent) x 2 (typical size difference: present or absent) design, defining 12 experimental conditions. The participants had to complete a total of 120 trials (12 experimental conditions x 10 trials).

We designed the experiment using the PsyScope software (Cohen, MacWhinney, Flatt, \& Provost 1993) and ran it on a Macintosh computer (G4 eMac). A chinrest was used to ensure that the distance to the screen remained constant (about $50 \mathrm{~cm}$ ).

\section{Procedure and design}

After filling in a written consent form, each participant was tested individually in a session that lasted approximately $10 \mathrm{~min}$. First, the instructions were presented on the screen. Next, the instructions were repeated orally to ensure that the participants had understood them well. The participants' task was to detect whether a picture (the target) was presented larger than the other objects, among two, six, or ten items displayed on the screen. Participants indicated their response by pressing the appropriate key on the keyboard, and the response keys were counterbalanced across participants. The participants began the experiment with ten practice trials featuring stimuli not used in the experimental trials.

A fixation point was presented for $1,000 \mathrm{~ms}$ at the start of each trial. Then, a blank screen was presented for $50 \mathrm{~ms}$ before the appearance of the display. The stimuli were displayed on the screen until the participant responded. A 2,000-ms intertrial interval separated the experimental trials.

\section{Results and discussion}

The mean correct response latencies and the mean percentages of correct responses were calculated across participants for each experimental condition. Latencies more than two standard deviations above or below the mean were excluded (less than $5 \%$ of the data). Analyses were performed on latencies and the rates of "present" responses, with participants as random variables, and number of items (two, six, or ten) and typical size difference (present or absent) as within- 
subjects factors. All of the statistical analyses were performed using STATISTICA (version 8.0, StatSoft, Inc.).

\section{Latencies}

Separate analyses of variance were performed for the presence or absence of the physical size difference.

When the physical size difference was present, a significant main effect of the number of items was observed $\left[F(2,70)=35.98, p<.05, \eta_{\mathrm{p}}^{2}=.51\right]$. The RTs required to detect the target became slower as the number of items increased. There was a main effect of the typical size difference $\left[F(1,35)=16.05, p<.05, \eta_{p}^{2}=.31\right]$, which indicated that RTs were shorter when the typical size difference was present than when it was absent. A significant interaction between typical size difference (absent or present) and number of items was observed $[F$ $\left.(2,70)=4.60, p<.05, \eta_{p}^{2}=.12\right]$. As Fig. 2 indicates, the influence of the number of items was less when typical size was present than when it was absent. Planned comparisons revealed an influence of typical size difference only in the ten-item condition $[F(1,35)=9.30, p<.05]$.

In summary, the main effect of typical size difference and the interaction between typical size difference and number of items allow us to conclude that the congruency between the typical and perceptual size difference led to faster responses in the visual search task.

When the physical size difference was absent, there was only a significant main effect of the number of items $[F(2$, $\left.70)=46.01, p<.05, \eta_{p}^{2}=.57\right]$; the main effect of typical size difference was not significant $[F(1,35)<1]$, nor was the interaction between typical size difference (present or absent) and the number of items $[F(2,78)=2.87, p=.06$; see Fig. 3]. These results suggest that there was no difference between the typical size difference conditions in terms of the perceptual quality of the stimuli. The participants saw the same targets in combination with the distractors in both

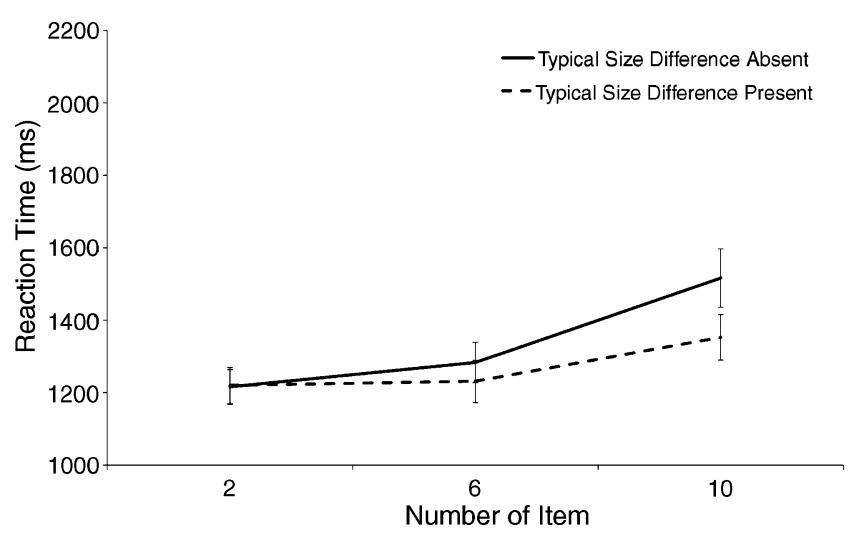

Fig. 2 Reaction times when physical size difference was present in Experiment 1, as a function of typical size difference and number of items. Vertical lines depict the standard errors of the means

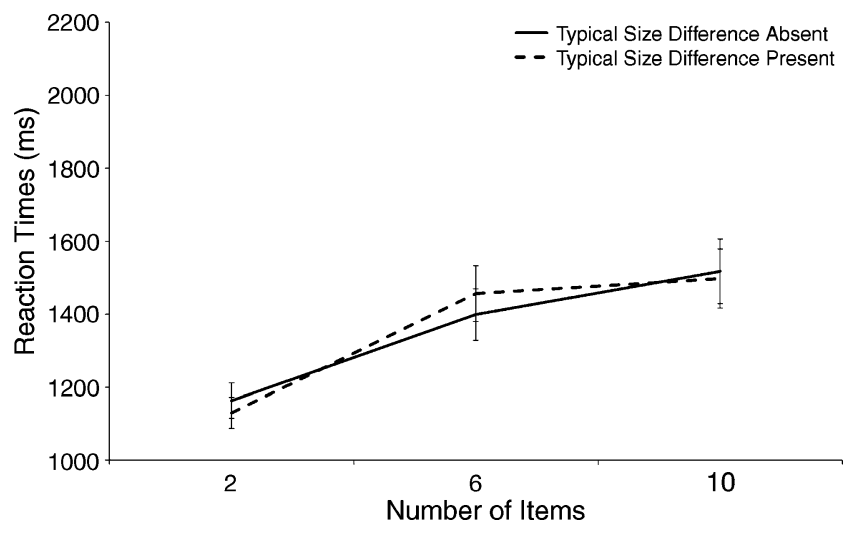

Fig. 3 Reaction times when physical size difference was absent in Experiment 1, as a function of typical size difference and number of items. Vertical lines depict the standard errors of the means

the conditions in which typical size difference was absent and present. In addition, the same target was also seen with the same distractors in the two physical size difference conditions (present or absent). If a perceptual difference between the two typical size difference conditions could explain the results when the physical size difference was present, then this effect should also have been observed when the physical size difference was absent. However, this was not the case.

\section{Rates of "present" responses}

When the physical size difference was present (see Table 1, top), we observed a significant main effect of the number of items $\left[F(2,70)=5.26, p<.05, \eta_{p}^{2}=.13\right]$. The main effect of the typical size difference was significant $[F(1,35)=$ 8.94, $\left.p<.05, \eta_{p}^{2}=.20\right]$. Whatever the number of items, participants had a tendency to answer "present" when typical size difference is present. The interaction between the number of items and the presence or absence of the typical size difference was significant $[F(2,70)=3.57, p<$ $\left..05, \eta_{p}^{2}=.09\right]$. Local comparisons indicated a significant difference in the ten-item condition $[F(1,35)=18.75, p<$ $.05]$. The ratio of "present" responses was greater when the typical size difference was present than when it was absent, and this effect grew as the number of items increased.

When the physical size difference was absent (see Table 1, bottom), no main effect of the number of items $[F(2,70)<1]$ or of the typical size difference $[F(1,35)=$ $3.02, p=.09]$ was observed. The interaction between the typical size difference (present or absent) and the number of items was significant $\left[F(2,70)=6.35, p<.05, \eta_{p}^{2}=.15\right]$ : The participants decided with greater frequency that the target was present when the typical size difference was present and with a larger number of items.

As we expected, the results clearly indicated that reactivation of the typical size difference during the 
Table 1 Means and standard errors for "present" response rates when physical (i.e., perceptual) size difference was present versus absent

\begin{tabular}{|c|c|c|c|c|c|c|}
\hline & \multicolumn{6}{|c|}{ Number of Items } \\
\hline & \multicolumn{2}{|l|}{2} & \multicolumn{2}{|l|}{6} & \multicolumn{2}{|l|}{10} \\
\hline & PR & $S E$ & PR & $S E$ & PR & $S E$ \\
\hline \multicolumn{7}{|l|}{ Physical Size Difference Present } \\
\hline Typical size difference present & .86 & .02 & .88 & .02 & .86 & .02 \\
\hline Absent & .83 & .02 & .85 & .02 & .76 & .02 \\
\hline \multicolumn{7}{|l|}{ Physical Size Difference Absent } \\
\hline Typical size difference present & .03 & .01 & .08 & .02 & .06 & .02 \\
\hline Absent & .04 & .01 & .03 & .01 & .02 & .02 \\
\hline
\end{tabular}

PR "present” response rate; $S E$ standard error. difference, the conditions were the same as those in Experiment 1.

For the physical size difference, the target could be displayed either as large as or smaller than the distractors. If the target was displayed smaller than the distractors, then physical size difference would be present. If the target was displayed as large as the distractors, then physical size difference would be absent.

It should be noted that the typical target size was still large and the physical size options were still 130 or 80 pixels. The typical size of the distractors could be large or small, but the physical size on the screen was always 130 pixels. The participants had to detect whether one picture (the target) was displayed smaller than the others (the distractors) on the screen.

\section{Results and discussion}

\section{Latencies}

The same separate analyses of variance were performed as in Experiment 1.

When the physical size difference was present, the main effect of number of items was significant $[F(2,70)=45.47$, $\left.p<.05, \eta_{p}^{2}=.56\right]$. The RTs required to detect the target became longer as the number of items increased. There was a main effect of the typical size difference $[F(1,35)=$ 27.74, $p<.05, \eta_{p}^{2}=.44$ ], indicating that RTs were shorter when the typical size difference was absent than when it was present. The interaction between typical size difference (absent or present) and number of items was not significant $[F(2,70)=1.80, p=.17$; see Fig. 4]. A post-hoc Tukey test revealed significant differences for two, six, and ten items when the typical size difference was either absent or present. The mean $\mathrm{RTs}$, respectively, were 1,134 and $1,232 \mathrm{~ms}$ for the two-item condition $(p<.05), 1,282$ and $1,460 \mathrm{~ms}$ for the six-item condition $(p<.05)$, and 1,365 and $1,490 \mathrm{~ms}$ for the ten-item condition $(p<.05)$.

These results show that RTs for the visual search were modulated by the presence of a (noncongruent) typical size

those described in Experiment 1. For the typical size 


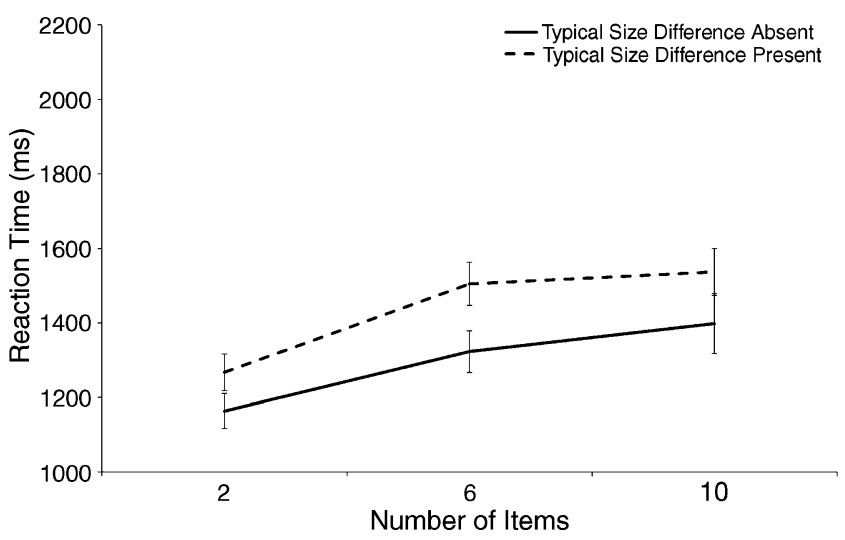

Fig. 4 Reaction times when physical size difference was present in Experiment 2, as a function of typical size difference and number of items. Vertical lines depict the standard errors of the means

difference with a smaller number of items than in Experiment 1 . This result was partially the reverse of the one in Experiment 1, since there were no interactions between the number of items and the typical size difference. This, however, might not be surprising when we think about search asymmetries (Treisman \& Gormican, 1988). In Experiment 2, searching for small targets among large distractors is a process known to be inefficient, and manipulating typical size might not have further impacted that inefficiency, although it did introduce a change in overall speed.

When the physical size difference was absent, only a significant effect of number of items was observed $[F(2,70)=$ $\left.226.80, p<.05, \eta_{p}^{2}=.86\right]$. There was no significant effect of typical size difference $[F(1,35)=1.56, p=.21]$ and no interaction between the presence or absence of typical size difference and the number of items $[F(2,70)<1$; see Fig. 5]. As in Experiment 1, these results suggest that there was no difference in the perceptual quality of the stimuli between the two conditions of the typical size difference.

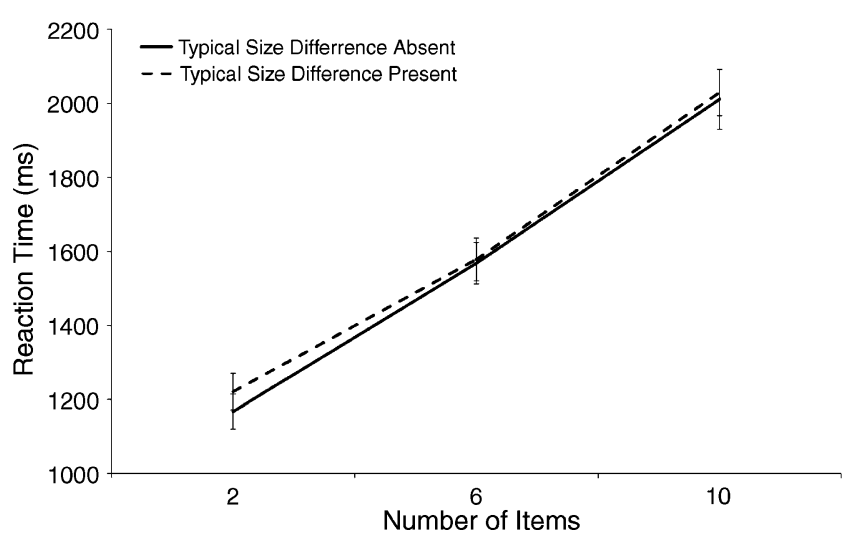

Fig. 5 Reaction times when physical size difference was absent in Experiment 2, as a function of typical size difference and number of items. Vertical lines depict the standard errors of the means
Rate of "present" responses

When the physical size difference was present (see Table 2, top), no significant main effect of number of items $[F(2,70)=2.93, p=.06]$ was observed. A significant main effect of typical size difference was revealed $\left[F(1,35)=7.49, p<.05, \eta_{p}^{2}=.17\right]$, but no interaction between the typical size difference and the number of items $[F(2,70)<1]$. The participants tended to answer "present" when the typical size difference was absent, irrespective of the number of items.

When the physical size difference was absent (see Table 2, bottom), no significant effect was observed. The analysis of variance revealed no main effect of the number of items $[F(1,35)<1]$, and the main effect of typical size difference did not reach threshold level $[F(1$, $35)=2.86, p=.06]$. The interaction between the typical size difference and the number of items was not significant $[F(2,70)=1.88, p=.16]$. These results are explainable, since when the typical size difference was present, it was not congruent with the visual search task, and the participants tended to respond "absent." When the typical size difference was absent, all of the objects had the same typical size, and the participants also responded "absent." In these two conditions, the participants responded "absent" with equal frequency but not for the same reasons.

As we expected, the addition of a noncongruent typical size difference disrupted responses in the visual search task.

\section{General discussion}

The aim of this research was to study the links between memory and perception. More precisely, we tested whether the addition of a typical size difference to a preexisting physical size difference would impact a visual search task. When the physical size difference was present, contrasting effects were observed in Experiment 1 and 2, depending on the congruency or noncongruency of the typical size difference with the physical size difference.

In Experiment 1, when the physical size difference was present, the main effect of typical size difference and the interaction between it and the number of items indicated that the RTs for visual search decreased when the typical size difference was present. These results suggest that the RTs were shorter when the typical size difference was congruent with the physical size difference. As for the rate of "present" responses, the participants answered "present" more frequently when the typical size difference was present than when it was absent. Consequently, Experiment 1 demonstrates that adding a congruent typical size difference to a physical size difference improves performance in the visual search task. 
Table 2 Means and standard errors for "present" response rates when physical (i.e., perceptual) size difference is present versus absent
$\mathrm{PR}$, "present" response rate; $S E$, standard error

\begin{tabular}{|c|c|c|c|c|c|c|}
\hline & \multicolumn{6}{|c|}{ Number of Items } \\
\hline & \multicolumn{2}{|l|}{2} & \multicolumn{2}{|l|}{6} & \multicolumn{2}{|l|}{10} \\
\hline & PR & $S E$ & PR & $S E$ & PR & $S E$ \\
\hline \multicolumn{7}{|l|}{ Physical Size Difference Present } \\
\hline Typical size difference present & .88 & .02 & .86 & .02 & .84 & .02 \\
\hline Absent & .91 & .01 & .90 & .02 & .88 & .01 \\
\hline \multicolumn{7}{|l|}{ Physical Size Difference Absent } \\
\hline Typical size difference present & .03 & .01 & .03 & .01 & .06 & .02 \\
\hline Absent & .04 & .01 & .02 & .01 & .04 & .01 \\
\hline
\end{tabular}

In contrast, in Experiment 2, when the physical size difference was present, the main effect of typical size difference indicated that the RTs for visual search increased when the typical size difference was present. These results indicate that the RTs were slower when the typical size difference was noncongruent with the physical size difference. The rate of "present" answers revealed that participants tended to answer "present" more frequently when the typical size difference was absent than when it was present. Consequently, Experiment 2 demonstrated that adding a noncongruent typical size difference to a physical size difference disrupted performance in the visual search task.

In Experiment 1 and 2, when the physical size difference was absent, no effect of typical size difference on RTs was above the threshold of significance. This result indicates that the perceptual quality of the stimuli was not different between the two typical size difference conditions. If a perceptual quality difference between the two conditions of typical size difference could explain the results when the physical size difference was present, this effect should have also been observed when the physical size difference was absent. However, this was not the case.

These results confirm our claim that memory dimensions are reactivated during perception (e.g., Brunel et al., 2009; Labeye, Oker, Badard, \& Versace 2008; Versace et al., 2009). Taken together, they suggest that memory and perception share resources. These results reveal an interaction between perceptual and memory dimensions that constitutes a further argument in support of a link between perceptual and memory mechanisms.

We argue that typical size has an effect on the computation of differences in the display. In these experiments, the participants searched for a target based on a perceptual difference (the physical size difference). A typical size difference was introduced. In both cases, the typical and perceptual differences were based on the same visual dimension: size. The perceptual difference between each set of the objects was computed in order to identify a stimulus that was either larger or smaller than the others.
According to the embodied cognition theory, the activation of the multisensory dimension in memory is defined as automatic and should occur during the perception process (see, e.g., Versace et al., 2009). This activation leads to a computation of the difference between typical dimensionshere, typical size-in response to the task demands. The typical size difference could be represented in a multidimensional similarity space (Nosofsky, 1991) in which the degree of similarity could be expressed as an exponential distance between the objects on the screen. In our study, the typical target size was always large. When the typical size difference was congruent, the distance between the target and the distractors was larger than when the typical size difference was noncongruent. At the same time, the participants were searching for a perceptual difference. This required them to compute the relative perceptual differences between each set of objects on the screen. We argue that the computation of typical size difference modulates the computation of perceptual size difference. The process of calculating perceptual or typical size difference seems to be shared between memory and perception.

However, the locus of the effect is still unknown. It is possible that the activation of the typical size difference results in competition or facilitation of responses when the typical size difference is, respectively, noncongruent or congruent with the physical size. As Predebon (1992; Predebon \& Woollen, 1994) has shown, familiar size has no effect on perception per se, but instead affects nonperceptual processes such as judgement. Alternatively, it is possible that the typical size difference may actually influence early perceptual processes, changing the perception of the object. Depending on the goals or expectations of the observers, activity in the early visual area can be modulated by topdown processes (e.g., Kastner, Pinsk, De Weerd, Desimone, \& Ungerleider 1999; O’Connor, Fukui, Pinsk, \& Kastner 2002). The present research does not allow us to know whether the effect we found occurs during the perception process or later during the decision process. More work would be needed to pinpoint the locus of this effect. 
Acknowledgement Thanks to Guillaume Vallet and Stéphane Rousset for their comments and several discussions. This research was supported by a Cifre grant from ANRT, ADIV, and ADN Marketing Science.

\section{References}

Barsalou, L. W. (1999). Perceptual symbol systems. The Behavioral and Brain Sciences, 22, 577-609. doi:10.1017/S0140525X99002149

Barsalou, L. W. (2003). Abstraction in perceptual symbol systems. Philosophical Transactions of the Royal Society B, 358, 11771187. doi:10.1098/rstb.2003.1319

Barsalou, L. W. (2005). Abstraction as dynamic interpretation in perceptual symbol systems. In L. Gershkoff-Stowe \& D. Rakison (Eds.), Building object categories (pp. 389-431). Mahwah: Erlbaum.

Barsalou, L. W. (2008). Grounded cognition. Annual Review of Psychology, 59, 617-645. doi:10.1146/annurev.psych.59. 103006.093639

Barsalou, L. W., Pecher, D., Zeelenberg, R., Simmons, W. K., \& Hamann, S. B. (2005). Multi-modal simulation in conceptual processing. In W. Ahn, R. Goldstone, B. Love, A. Markman, \& P. Wolff (Eds.), Categorization inside and outside the lab: essays in honor of Douglas L. Medin (pp. 249-270). Washington, DC: American Psychological Association.

Brunel, L., Labeye, E., Lesourd, M., \& Versace, R. (2009). The sensory nature of episodic memory: Sensory priming effects due to memory trace activation. Journal of Experimental Psychology. Learning, Memory, and Cognition, 35, 1081-1088. doi:10.1037/ a 0015537

Cohen, J. D., MacWhinney, B., Flatt, M., \& Provost, J. (1993). PsyScope: a new graphic interactive environment for designing psychology experiments. Behavior Research Methods, Instruments, \& Computers, 25, 257-271.

Hansen, T., Olkkonen, M., Walter, S., \& Gegenfurtner, K. R. (2006). Memory modulates color appearance. Nature Neuroscience, 3, 1367-1368. doi:10.1038/nn1794

Howard, R. J., Ffytche, D. H., Barnes, J., McKeefry, D., Ha, Y., Woodruff, P. W., et al. (1998). The functional anatomy of imagined and perceived colour. NeuroReport, 9, 1019-1023.

Kastner, S., Pinsk, M. A., De Weerd, P., Desimone, R., \& Ungerleider, L. G. (1999). Increased activity in human visual cortex during directed attention in the absence of visual stimulation. Neuron, 22, 751-761. doi:10.1016/S0896-6273(00)80734-5

Labeye, E., Oker, A., Badard, G., \& Versace, R. (2008). Activation and integration of motor components in a short-term priming paradigm. Acta Psychologica, 129, 108-111. doi:10.1016/j. actpsy.2008.04.010

Martin, A., \& Chao, L. L. (2001). Semantic memory and the brain: Structure and processes. Current Opinion in Neurobiology, 11, 194-201. doi:10.1016/S0959-4388(00)00196-3
Martin, A., Haxby, J. V., Lalonde, F., Wiggs, C. L., \& Ungerleider, L. G. (1995). Discrete cortical regions associated with knowledge of color and knowledge of action. Science, 270, 102-105.

Nosofsky, R. M. (1991). Test of an exemplar model for relating perceptual classification and recognition memory. Journal of Experimental Psychology: Human Perception and Performance, 17, 3-27.

O’Connor, D. H., Fukui, M. M., Pinsk, M. A., \& Kastner, S. (2002) Attention modulates responses in the human lateral geniculate nucleus. Nature Neuroscience, 5, 1203-1209. doi:10.1038/nn957

Paulesu, E., Harrison, J., Baron-Cohen, S., Watson, J. D. G., Goldstein, L., Heather, J., et al. (1995). The physiology of coloured hearing: A PET activation study of colour-word synaesthesia. Brain, 118, 661-676.

Pecher, D., Zeelenberg, R., \& Barsalou, L. W. (2003). Verifying properties from different modalities for concepts produces switching costs. Psychological Science, 14, 119-124. doi:10.1080/03640210802035365

Predebon, J. (1992). The influence of object familiarity on magnitude estimates of apparent size. Perception, 21, 77-90.

Predebon, J., \& Woollen, S. J. (1994). The familiar-size cue to depth under reduced-cue viewing conditions. Perception, 23, 1301-1312.

Slotnick, S. D. (2004). Visual memory and visual perception recruit common neural substrates. Behavioral and Cognitive Neuroscience Reviews, 3, 207-221. doi:10.1177/1534582304274070

Slotnick, S. D., \& Schacter, D. L. (2006). The nature of memory related activity in early visual areas. Neuropsychologia, 44, 2874-2886. doi:10.1016/j.neuropsychologia.2006.06.021

Spence, C., Nicholls, M. E. R., \& Driver, J. (2001). The cost of expecting events in the wrong sensory modality. Perception \& Psychophysics, 63, 330-336.

Treisman, A. M., \& Gormican, S. (1988). Feature analysis in early vision: Evidence from search asymmetries. Psychological Review, 95, 1548.

Vallet, G., Brunel, L., \& Versace, R. (2010). The perceptual nature of the cross-modal priming effect. Experimental Psychology, 57, 376-382. doi:10.1027/1618-3169/a000045

Van Dantzig, S., Pecher, D., Zeelenberg, R., \& Barsalou, L. W. (2008). Perceptual processing affects conceptual processing. Cognitive Science, 32, 579-590. doi:10.1080/03640210802035365

Vermeulen, N., Corneille, O., \& Niedenthal, P. M. (2008). Sensory load incurs conceptual processing costs. Cognition, 109, 287294. doi:10.1016/j.cognition.2008.09.004

Vermeulen, N., Mermillod, M., Godefroid, J., \& Corneille, O. (2009). Unintended embodiment of concept into percepts: Sensory activation boosts attention for same-modality concepts in the attentional blink paradigm. Cognition, 112, 467-472. doi:10.1016/j.cognition.2009.06.003

Versace, R., Labeye, E., Badard, G., \& Rose, M. (2009). The contents of long-term memory and the emergence of knowledge. European Journal of Psychology, 21, 522-560. doi:10.1080/ 09541440801951844

Weinberger, N. (2004). Specific long-term memory traces in primary auditory cortex. Nature Neuroscience, 5, 279-290. doi:10.1038/ nrn1366 